\title{
Novel Approach of Linear Accelerator-Based Stereotactic Radiosurgery in an Adult Patient With Brain Arteriovenous Malformation: A Case Report and Clinical Outcome
}

\author{
Santosh Devarakonda ${ }^{10}$, Vijay Kumar Kontham ${ }^{1 *}$ \\ ${ }^{1}$ Department of Radiation Oncology, Apple Cancer Institute, Apple Hospitals and Research Institute Ltd, Kadamwadi, \\ Kolhapur, MH, India.
}

\begin{abstract}
Stereotactic radiosurgery (SRS) is a proven strategy for treating the central nervous system arteriovenous malformation (AVM). We intend to present a case of cerebral AVM in an adult patient, successfully treated with a linear accelerator (LINAC) based SRS using simplified setup techniques and an advanced planning system. In the current scenario, frame-based SRS is the standard of care with LINAC. Recently, it has proven that even frameless SRS has similar results. In our case, we used a frameless SRS technique with similar results. While calculating the dose in radiotherapy planning, we used the Acuros ${ }^{\mathrm{TM}}$ algorithm, which has proven dosimetric advantages compared to the traditional AAA ${ }^{\mathrm{TM}}$ algorithm.

Keywords: Case report; Stereotactic radiosurgery; Arteriovenous malformation; Acuros; Radiotherapy.
\end{abstract}

\author{
*Correspondence to \\ Vijay Kumar Kontham, \\ Department of Radiation \\ Oncology, Apple Cancer \\ Institute, Apple Hospitals \\ and Research Institute Ltd, \\ Kadamwadi, Kolhapur, $\mathrm{MH}$, \\ India. \\ Email: vijai2k6@gmail.com
}

Published online 28 September, 2020

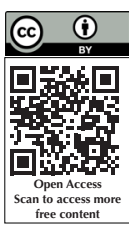

Citation: Devarakonda S, Kontham VK, Novel Approach of Linear Accelerator-Based Stereotactic Radiosurgery in an Adult Patient With Brain Arteriovenous Malformation: A Case Report and Clinical Outcome. Clin Neurosci J. 2020;7(4):219-221. doi:10.34172/ icnj.2020.29.

\section{Introduction}

Central nervous system arteriovenous malformations (AVMs) are relatively rare developmental anomalies for both pediatric and adult populations. It is although not very rare to treat these AVMs with radiosurgery. ${ }^{1}$ There have been multiple case series published in the literature about the possible treatment options and stereotactic radiosurgery (SRS) role. Gamma Knife, CyberKnife, LINAC based SRS, and proton beam radiosurgery are different options used for the delivery of SRS. ${ }^{1}$ Frameless SRS has been proven to be equally effective as framebased SRS in clinical practice. ${ }^{2}$ We describe a successful attempt of SRS in an adult female with cerebral AVM.

\section{Clinical Presentation}

A 28-year-old young female, with no medical comorbidities, was presented to our radiation oncology outpatient services. A neurosurgeon referred her. She complained of frequent episodes of headaches for the past one year, of which she was responding to medication. There were no symptoms of a neurological deficit.

\section{Final Diagnosis}

On MR (magnetic resonance) imaging (Figure 1), a large lobulated tangle of vascular flow voids has seen involving left high frontoparietal brain parenchyma near the surface. On MR angiogram, Left peri-central gyri superficial pial AVM has diagnosed. The nidus approximately measured $2.2 \times 1.4 \times 1.8 \mathrm{~cm}$ in size, with feeding arteries from the middle cerebral artery and a small branch from the anterior cerebral artery. There are prominent draining veins into superior sagittal sinus. The lesion is SpetzlerMartin AVM grade II (small nidus with the eloquentsensory motor area). The patient refused the option of surgery due to the risk of potential complications.

\section{Treatment}

This patient has booked for SRS treatment on our Varian $^{\text {Truebeam }}{ }^{\text {TM }}$ STx $^{\text {TM }}$ LINAC with HD MLC (high definition multi-leaf collimators) and FFF (flattening filter free) configuration. We have used simplified setup and immobilization techniques for this patient. A total of 3 clamp head immobilization thermoplastic mask were prepared with suitable headrest and aligned the patient with couch axis using room mounted laser setup. The CT isocentre was marked using collimator rotations at 0 and 45-degree angles and gantry rotations of 0,90 , and 270 degrees. A planning CT scan with IV contrast was taken with $0.6 \mathrm{~mm}$ slice thickness and imported to the treatment planning system. The CT images have

(C) 2020 The Author(s). This is an open access article distributed under the terms of the Creative Commons Attribution License (http:// creativecommons.org/licenses/by/4.0/), which permits unrestricted use, distribution, and reproduction in any medium, provided the original work is properly cited. 
registered with MR images, and target volumes and organs at risk were delineated. A marginal dose of 18 Gy has prescribed to planning target volume (PTV). Planning has done with Acuros ${ }^{\mathrm{Tm}}$ algorithm. The gross volume of nidus received $100 \%$ of the dose to $100 \%$ volume with planning target volume receiving $98 \%$ of the dose to $98 \%$ volume. Brain-PTV received 8.75 Gy to $12 \mathrm{cc}$ and 9.66 Gy to $10 \mathrm{cc}$ volumes, which were under normal tolerance limits. There was a $50 \%$ dose fall off within $5 \mathrm{~mm}$ outside PTV margin. The treatment has administered in a single fraction after meticulous QA and setup of patients with verification of Manual and automated SSD values at representative angles. Pre- and post-treatment cone beam computed tomography (CBCT) has taken to confirm sub-mm precision. Plan parameters: Maximum tumour dimension- $2.6 \mathrm{~cm}$, volume- $9.4 \mathrm{cc}$, conformity index 3.01 and Homogeneity Index 0.11. Gradient Index 2.7.

\section{Outcome and Follow-up}

On follow up at 12 months, the patient improved symptomatically, and MR imaging (Figure 2) revealed a decrease in the size of AVM nidus, measuring approximately $1.3 \times 1.0 \times 1.5 \mathrm{~cm}$. At 24 months, MR with angiography revealed a complete resolution of AVM

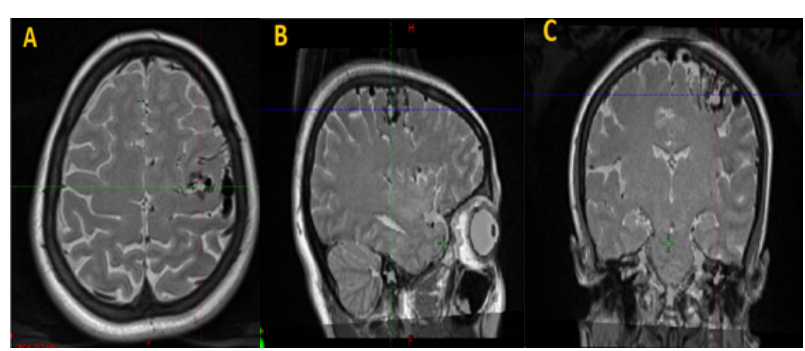

Figure 1. Pre-Treatment. Axial, Sagittal and Coronal Images of T2 MRI Depicting AVM (Accessed on 05 $5^{\text {th }}$ June, 2020. Available from patient's case file; Scanned in February 2018).

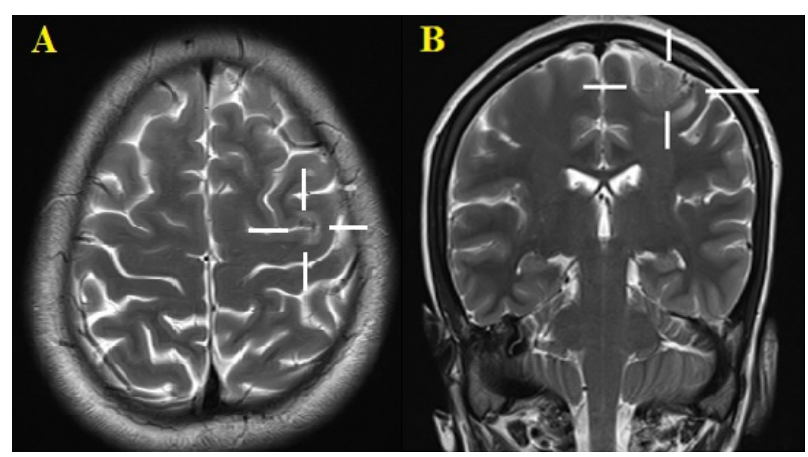

Figure 2. Post-Treatment. Axial and Coronal Sections of T2 MRI Images Taken 24 Months Post SRS-Depicting Complete Obliteration of AVM Nidus. (Accessed on 05th June, 2020. Available from patient's case file; Scanned in March 2020) nidus.

\section{Discussion}

Central nervous system AVM poses a significant challenge for the clinicians, as it requires skill and intuition for optimal management. Inoperable or small AVMs and those in eloquent regions are indications for SRS in most of the patients. The treatment of brain AVM's with LINAC based SRS is equivalent to other modalities like Gamma knife and CyberKnife based radiosurgery. ${ }^{1}$ Treatment outcomes were comparable in both pediatric and adult populations. Age seems to be a poor predictor of outcome. SRS is a reliable treatment option for brain AVMs across the ages. ${ }^{3}$ Frameless SRS is an effective and non-invasive approach, typically using Brainlab immobilization setup with three-layered masks. ${ }^{4}$ In contrast, we used a simplified approach for patient setup with a simple singlelayered thermoplastic mask with a high degree of patient repositioning accuracy. Obliteration of AVMs after SRS has been reported to range from $35 \%$ to $92 \%$, with the obliteration rate exceeding $70 \%$ in most series. ${ }^{1}$ The obliteration rate with small AVMs has exceeded $80 \%$ in most series. ${ }^{5,6}$

The most important predictor of the obliteration of AVM was a higher marginal dose of radiation. Potts et $\mathrm{al}^{7}$ reported a $52 \%$ rate of obliteration with a dose $>18$ Gy, while only $16 \%$ of AVMs that received $<18$ Gy has obliterated in a series reporting on the results of GKbased SRS for AVMs.

The interval-to-obliteration after SRS could range from 1 to 4 years or even longer. ${ }^{8}$ The new onset of neurological deficits after SRS has reported in $0 \%-17.6 \%$ of patients in different series of LINAC- and GK-based SRS in AVMs. Permanent neurological deficits have found after SRS has reported occurring in $1.5 \%-6 \%$ of patients. A higher incidence of radiation-induced complications has reported in children with larger volumes of AVM, Spetzler-Martin grade IV and V AVMs, and those located in the brainstem, thalamus, or basal ganglia. ${ }^{1}$ A practical, clinically oriented goal in the management of intracranial AVMs is the prospective avoidance of neurological sequelae, most commonly manifesting in stroke or even death. ${ }^{9}$ While deep-seated AVMs are more likely to rupture during the latency period post SRS, the driving factor for the reduction of hemorrhage risk is the obliteration of AVM. ${ }^{10}$ Our patient did not develop any event of neurological deficit or intracranial hemorrhage during post SRS follow up. Traditionally, AAA $^{\mathrm{Tm}}$ algorithm would have used for SRS planning on Varian LINACs, but in our center, we used the Acuros ${ }^{\mathrm{Tm}}$ algorithm for dose calculation. Compared to $\mathrm{AAA}^{\mathrm{m}}$ algorithm, plans calculated with Acuros ${ }^{\mathrm{Tw}}$ algorithm have a lower conformity index and higher homogeneity index, D1\% PTV (dose to 1\% PTV volume), and R50 (ratio of 
$50 \%$ of prescription isodose volume to PTV). Although these differences are clinically less significant, Acuros ${ }^{\mathrm{Tm}}$ improves the accuracy of radiotherapy dose calculation. ${ }^{11}$

\section{Conclusion}

Our case report intends to show the possibility of executing frameless SRS even without designated immobilization devices. With more straightforward and innovative techniques, it is possible to achieve highly accurate results. In the Varian platform, though $\mathrm{AAA}^{\mathrm{mm}}$ and $\mathrm{Acuros}^{\mathrm{Tm}}$ offer similar clinical benefits, Acuros ${ }^{\mathrm{Tm}}$ delivers a higher level of dosimetric advantages.

\section{Conflict of Interest}

The authors declare that they have no conflict of interest.

\section{Authors' Contribution}

SD the patient's radiation oncologist, reviewed the literature, and contributed to manuscript drafting. VKK contributed to preparing the draft and collecting all sources of data.

\section{Ethical Statement}

Informed written consent before treatment and before publication of case report was taken from the patient. Institutional Ethical committee approval was taken. Patient anonymity maintained. Authors take comprehensive responsibility of the research. No offensive, discriminatory, or other unacceptable language was used.

\section{References}

1. Moorthy RK, Rajshekhar V. Stereotactic radiosurgery for intracranial arteriovenous malformations: a review. Neurol India. 2015;63(6):841-51. doi: 10.4103/0028-3886.1701

2. Chen AY, Hsieh Y, McNair S, Li Q, Xu KY, Pappas C. Frame and frameless linear accelerator-based radiosurgery for idiopathic trigeminal neuralgia. J Radiosurg SBRT. 2015;3(4):259-70.

3. Chen CJ, Ding D, Kano H, Mathieu D, Kondziolka D, Feliciano
C, et al. Stereotactic radiosurgery for pediatric versus adult brain arteriovenous malformations. Stroke. 2018;49(8):193945. doi: 10.1161/strokeaha.118.022052.

4. Minniti G, Scaringi C, Clarke E, Valeriani M, Osti M, Enrici RM. Frameless linac-based stereotactic radiosurgery (SRS) for brain metastases: analysis of patient repositioning using a mask fixation system and clinical outcomes. Radiat Oncol. 2011;6:158. doi: 10.1186/1748-717x-6-158.

5. Bowden G, Kano H, Caparosa E, Tonetti D, Niranjan A, Monaco EA, 3rd, et al. Stereotactic radiosurgery for arteriovenous malformations of the postgeniculate visual pathway. J Neurosurg. 2015;122(2):433-40. doi: 10.3171/2014.10. jns1453.

6. Burrow AM, Link MJ, Pollock BE. Is stereotactic radiosurgery the best treatment option for patients with a radiosurgery-based arteriovenous malformation score $\leq 1$ ? World Neurosurg. 2014;82(6):1144-7. doi: 10.1016/j.wneu.2014.07.009.

7. Potts MB, Sheth SA, Louie J, Smyth MD, Sneed PK, McDermott MW, et al. Stereotactic radiosurgery at a low marginal dose for the treatment of pediatric arteriovenous malformations: obliteration, complications, and functional outcomes. J Neurosurg Pediatr. 2014;14(1):1-11. doi: 10.3171/2014.3.peds13381.

8. Zacest AC, Caon J, Roos DE, Potter AE, Sullivan T. LINAC radiosurgery for cerebral arteriovenous malformations: a single centre prospective analysis and review of the literature. J Clin Neurosci. 2014;21(2):241-5. doi: 10.1016/j. jocn.2013.03.040.

9. Tonetti DA, Gross BA. Re-evaluating clinical outcomes for AVM stereotactic radiosurgery. Prog Neurol Surg. 2019;34:267-72. doi: 10.1159/000493073.

10. Ding D, Chen CJ, Starke RM, Kano H, LeeJYK, Mathieu D, et al. Risk of brain arteriovenous malformation hemorrhage before and after stereotactic radiosurgery. Stroke. 2019;50(6):138491. doi: 10.1161/strokeaha.118.024230.

11. Mynampati D, Kuo H, Yaparpalvi R, Scripes PG, Tome W. SUE-T-122: Anisotropic analytical algorithm (AAA) vs. Acuros $\mathrm{XB}(\mathrm{AXB})$ in stereotactic treatment planning. Med Phys. 2015;42(6 Pt 13):3359-. doi: 10.1118/1.4924483. 